\title{
Cómo no impedir que los niños aprendan inglés
}

\author{
Javier Zanón
}

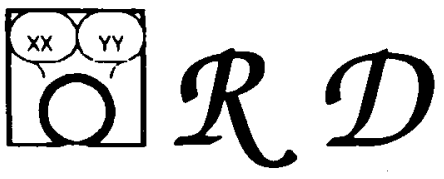

Se revisan en este artículo los problemas que un enfoque comunicativo basado en el adulto presentan para el diseño curricular del inglés para niños y se propone pasar a un diseño basado en actividades (más que en contenidos lingüisticos), alertando a la vez de las condiciones (que boy no se cumplen) necesarias para aplicarlo con eficacia, como la formación de los docentes o los recursos en el centro.

\section{INTRODUCCION}

¿Cómo enseñar inglés a niños y niñas de ocho años? Mediante un método comunicativo, dirán algunos. Sin embargo, la interpretación clásica de este término ha llevado a que la mayoría de cursos comunicativos para niños sean versiones simplificadas $\mathrm{y} / \mathrm{o}$ reducidas de un sistema de trabajo concebido y diseñado para adultos. De esta manera, encontramos que el patrón «Enfoque Comunicativo» (Zanón, 1988/89) se ha adaptado para cubrir las necesidades y características del público infantil. Desgraciadamente, los criterios utilizados para realizar estas adaptaciones no parecen basarse en consideraciones didácticas, y mucho menos parecen tener en cuenta las necesidades de los niños de ocho años, arrojando resultados muy desiguales.

Dado que en nuestro contexto educativo la aplicación de estos cursos parece ser la fuente más utilizada y que la edad mayoritaria de comienzo suele ser los ocho años, parece interesante preguntarse por las condiciones que desde una perspectiva psicopedagógica debería cumplir el diseño de cursos de inglés para niños y niñas de ocho años.

Para ello: I. Describiremos y analizaremos las características de los denominados «cursos comunicativos» de inglés para niños. Lo que nos llevará a alertar a los lectores sobre una serie de peligros intrínsecos al diseño de estos cursos y que pueden impedir el aprendizaje de la lengua extranjera. II. En segundo lugar, presentaremos un conjunto de principios de naturaleza psicolingǘstica que determinan el diseño y a secuenciación de actividades para el aprendizaje de una lengua extranjera a los ochos años. III. En tercer lugar, propondremos un sistema de trabajo alternativo, un sistema centrado en las actividades reali- 
zadas por niños y niñas y no en los contenidos lingüísticos objeto de aprendizaje. IV. Finalmente, reflexionaremos sobre las consecuencias de la adopción de un sistema de enseñanza del inglés basado en actividades dedicando una especial atención a la situación actual del profesorado en nuestro país y a sus necesidades de formación.

\section{LOS CURSOS COMUNICATIVOS PARA NIÑOS}

\section{Estructura del sistema de trabajo}

Una mirada a nuestras escuelas nos muestra el sistema de trabajo utilizado con más frecuencia. En su núcleo, un libro de texto «comunicativo» con la etiqueta «English for young learners» que determina la programación de contenidos. Esta se articula en unidades didácticas (Fig. 1) que ofrecen un cómodo progreso por un listado de temas de comunicación (los animales, tiendas y lugares en la ciudad, etc.) con los que se trabajan una serie de funciones comunicativas (dar información sobre cómo llegar a un sitio, decir de quién son las cosas, hablar sobre la gente, etc.) que, a su vez, generan una serie de muestras («Turn right/turn left») y estructuras («Possessives: his/her») lingüiticas para cada unidad.

\section{FIGURA 1}

\begin{tabular}{|c|c|c|c|}
\hline Unit & & Language items & Functions and topics \\
\hline 8 & page 29 & $\begin{array}{l}\text { Have you got your bag? } \\
\text { Yes, I bave/No, I baven't } \\
\text { Has she got her umbrella? } \\
\text { Yes, she bas/No, she basn't } \\
\text { Adjectives: tall, short, etc. }\end{array}$ & Asking for information \\
\hline 9 & page 33 & $\begin{array}{l}\text { Can you see an elephant? } \\
\text { Yes, I can/No, I can't } \\
\text { Whose cage is this? } \\
\text { Possessive-'s: the lion's cage. } \\
\text { Turn right, turn left. }\end{array}$ & $\begin{array}{l}\text { Talking about ability } \\
\text { Talking about possession } \\
\text { Zoo animals } \\
\text { Giving directions }\end{array}$ \\
\hline 10 & page 37 & $\begin{array}{l}\text { Show me the green kite. } \\
\text { What colour is her skirt? } \\
\text { It's red. } \\
\text { Revision. }\end{array}$ & $\begin{array}{l}\text { Colours } \\
\text { Clothes }\end{array}$ \\
\hline 11 & page 41 & $\begin{array}{l}\text { He's got brown legs } \\
\text { He's from England }\end{array}$ & $\begin{array}{l}\text { Parts of the body } \\
\text { Saying where people or things } \\
\text { come from }\end{array}$ \\
\hline 12 & page 45 & $\begin{array}{l}\text { Plural nouns: -_s/-es. } \\
\text { There's.../There are... } \\
\text { Is there..? } \\
\text { Yes, there is/No, there isn't. } \\
\text { Prepositions: in, on. }\end{array}$ & $\begin{array}{l}\text { Describing situations } \\
\text { Asking for information } \\
\text { Specifiging location }\end{array}$ \\
\hline
\end{tabular}

Ejemplo de syllabus comunicativo (reproducido de Strange 1989: p. 10)

De esta forma el syllabus se organiza como un listado de contenidos seccionado en lecciones. Cada una de las lecciones estructuradas sobre estas tres dimensiones de contenidos se convierten en trabajo en el aula mediante una serie de actividades que se caracterizan por organizarse en tres estadios: 
I. Un primer grupo de actividades de presentación de las estructuras/funciones («Possessives: saxon genitive» + «hablar sobre pertenencia») objeto de la lección. Para ello se utiliza un tema considerado «infantil» («Zoo animals») donde niños-personajes (normalmente personajes fijos en el libro) hacen las cosas propias del tema (ir al zoo) utilizando las funciones y estructuras de la lección mediante muestras («this is the lion's cage») insertadas en la historia. La presentación incluye una explotación de la historia en forma de actividades variadas: preguntas de comprensión, ejercicios de repetición, extracción de vocabulario, etc.).

II. Un segundo estadio de actividades de aprendizaje. Aunque puede adoptar diferentes formas (repetición en coro, juegos de simulación, ejercicios de substitución, etc.) su esencia es la de separar una a una cada estructura y/o función y hacer que los niños las reproduzcan mediante la realización de ejercicios (fig. 2). De esta manera se pretende el dominio aislado de cada uno de los ítems presentes en el índice de la lección.

\section{Figura 2}

\section{STAGE 1}

Divide the class into groups of three or four pupils Ask each group to hold up the mask of one of the characters and identify him her. To do this, stand near each group in turn and give a model. e. g. This is Carol.

\section{STAGE 2}

(a) Ask the pupils to look at the photograph of Carol and her family. Draw a rough sketch of it on the board. The characters can easily be identified by their position in the photograph and their height. This can be also done on the magnetboard/flannelgraph with the character cutouts at the back of this book. Say: This is Carol. This is Carol's brother. (CR) Then talk about all the members of Carol's family in the same way, e. g. This is Carol's....
Then go on to the photograph of Pat's family.

Follow the same procedure Say: This is

Pat. This is Pat's sister. This is Pat's... etc.

(b) Pick up the mask of Andrew and show it to the class. Say e. g.:

\section{T Show me Andrew's sister.}

The pupils will have to pick up the mask of Carol, hold it up and say:

\section{P1 This is Andrew's sister.}

To convey the meaning of Show me mime the action or translate. Continue in the same way, e. g.

$\mathrm{T}$ Show me Becky's sister.
Michael's sister.
Pat's sister.
Andrew's sister.
Michael's brother.

Ejemplo de actividad de aprendizaje (reproducido de Concari et al., 1983:14)

III. Finalmente, en una tercera fase se intentan actividades de ejercitación. Es decir, aquello aprendido para y con personajes de libro ahora lo utilizaremos en referencia a las cosas y la realidad de nuestros alumnos. De esta manera se practican las estructuras y funciones para comunicar sobre aspectos reales (Fig. 3). Aquí, las actividades adoptarán formas muy diversas (describir la propia familia, colorear un dibujo, medir y apuntar las alturas de los niños, etc.), pero siempre buscando la ejercitación de lo aprendido en la fase anterior. 
Figura 3

\section{STAGE 3}

Ask three or four pupils to go to the board, write the names of their brothers and/or sisters and then sit down. Tell the rest of the class that now you are going to see if you remember who is whose sister/brother. Give the first example yourself. Say:

\section{$\mathrm{T} X$ is Y's brother.}

Tell the pupils that when somebody gives the wrong relationship, the others in the class should correct him. e. g.

\section{P1 No. $X$ is Z's brother.}

Ejemplo de actividad de ejercitación (reproducido de Concari et al., 1983:15)

A estos tres estadios de elaboración de los contenidos cabría añadir un cuarto encarnado en los apartados de revisión que suelen aparecer en las programaciones cada tres o cinco unidades didácticas. En este caso, se recopilan los contenidos clave de cada unidad para ser repasados mediante una serie de ejercicios, generalmente sin nexo entre sí y a razón de un ejercicio por cada contenido a revisar. Sospechosamente, la mayoría de estos ejercicios tienen como objetivo comprobar la corrección gramatical de la estructura enseñada, obviando otro tipo de actividad más centrada en la práctica de la comunicación.

Además, desde este sistema de trabajo, los/as perofesores/as disponemos de abundantes materiales de apoyo (cuaderno de ejercicios, cuaderno de dibujo, libro de canciones, libro de actividades, etc.) diseñados paralelamente al curso central y que ofrecen un sinfín de posibilidades de acompañamiento para cada una de las unidades de que consta el curso. De esta manera se ofrece una mayor variedad de ejercicios (con actividades de plástica, con canciones, con juegos, etc.) que contemplan los contenidos objeto de trabajo de cada unidad, lo que facilita la adaptación a las características del profesor y los alumnos.

Como veremos a continuación, este planteamiento didáctico conlleva graves peligros para el aprendizaje del inglés. Haciendo que su utilizáción, más que enseñar, pueda impedir que niños y niñas aprendan inglés. Aunque todos sabemos que ningún profesor utiliza un curso como los esquematizados siguiéndolo al ciento por ciento, la estructura descrita condiciona los resultados del sistema de trabajo (en términos de qué inglés aprenden nuestros niños y niñas) al margen de las posibles modificaciones, adiciones o adaptaciones que los/as profesores/as más experimentados podamos hacer.

\section{Problemas de los cursos comunicativos}

Esto se debe a cuatro problemas fundamentales: i) La falta de un análisis de las necesidades y posibilidades de comunicación en lengua extranjera de niñas y niños de ocho años. II. El concepto de contenidos comunicativos reducido a estructuras lingüísticas, nociones y funciones. III. La concepción adulta de la organización del curso, con un sistema de secuenciación lineal de contenidos en unidades y de progreso en estadios de elaboración de los mismos dentro 
de cada unidad. IV. La ausencia de criterios psicolingüísticos acordes a la edad de ocho años para el diseño de actividades.

Los cursos descritos ofrecen una programación «comunicativa» cuyas unidades se derivan de los cursos nocional-funcionales desarrollados a partir de los trabajos del Consejo de Europa (Trim, 1973; Van ek, 1975). Un primer punto sobre el que prestar nuestra atención. ¿Podemos considerar adecuados para niños contenidos y materiales desarrollados desde un sistema de enseñanza diseñado para adultos?

Un análisis más detallado nos da una respuesta inmediata. Las programaciones nocional-funcionales se derivan de un análisis de las necesidades de comunicación de un tipo medio de aprendiz adulto que necesita el inglés en situaciones profesionales o de contacto con otros adultos que hablan en inglés. De esta manera se generan un conjunto de tópicos de comunicación (la identificación personal, los planes futuros, la obtención de información sobre viajes, etc, que anclados en situaciones precisas (la agencia de viajes, la calle, la entrevista, etc) representan el núcleo de necesidades de comunicación de los aprendices. El desarrollo de éstos en forma de contenidos lleva a especificar qué funciones (pedir información sobre horarios y precios de transportes, narrar sucesos pasados, saludar, etc.), nociones (la división horaria del día en GB, etc.), y contenidos gramaticales (preguntas con Wh-, uso de ago", etc.). De esta manera se obtienen unidades didácticas de gran precisión que reconstruyen el camino hacia las necesidades comunicativas de los aprendices. De los aprendices adultos, se nos olvidaba decir. Y ahí es donde se encuentra la piedra de toque de esta primera argumentación.

Desgraciadamente, la elaboración de programaciones comunicativas para niños se ha limitado a reproducir este esquema, si se me permite «infantilizándolo». En efecto, los programas de los cursos son meros cursos nocional-funcionales a los que se ha quitado todo aquello «demasiado adulto» o en el mejor de los casos se ha realizado uıı adaptación (casi siempre con personajes infantiles que hacen y dicen las cosas) a situaciones más próximas al mundo infantil. Este hecho conlleva resultados sorprendentes. Materiales didácticos donde los personajes «van al teatro» y los aprendices deben identificarse con la situación o ejercicios consistentes en dar instrucciones para indicar dónde está la calle tal o la tienda cual. Nada más próximo al mundo comunicativo del niño de ocho años.

A esta falta de adecuación de los aspectos temáticos de los contenidos, se suma un grave error de concepto. La selección de contenidos mediante estructuras, nociones y funciones reduce lo que se va a aprender a un catálogo de unidades conceptuales, como si de enseñar historia se tratara. Es decir, se trata el lenguaje como una materia de contenidos conceptuales, cuando precisamente es todo lo contrario. Una materia de contenidos instrumentales, donde los que se aprende se demuestra haciendo, no diciendo cosas sobre la materia (como también pasa en otras áreas como el dibujo, la música, etc.). De esta manera se olvida que se trata de enseñar a hablar, leer, escribir y entender en inglés, reduciendo el diseño del curso a un programa de acumulación de contenidos.

Además, la organización y tratamiento de los contenidos no tiene nada que ver con la «realidad» del uso de esos contenidos por los niños. La única manera de aprender a hablar y entender inglés es diciendo y escuchando cosas en inglés. La única manera de que esos contenidos sean potencialmente utilizables para comunicar es que se sitúen en el contexto aula. Para ello, deben ser contenidos 
que vayan a ser utilizados para hacer cosas con ellos en las actividades de la clase de inglés.

Como hemos visto, las programaciones comunicativas se limitan a presentar a los niños una serie de muestras lingüísticas y en el mejor de los casos a repetirlas/memorizarlas sin que se creen en el aula verdaderos espacios de comunicación. Esto además se realiza de forma lineal. Lo ya visto no vuelve a aparecer o si lo hace es para ser meramente recordado. Es decir, se ha aplicado un esquema de elaboración de contenidos exclusivamente adulto. La adición una a una de las unidades de contenido permitirán su aprendizaje lógico, organizando el material en torno a criterios de necesidades adultas (aquí los saludos y expresiones sociales, acá las diferentes formas de hablar del futuro, allá el funcionamiento de comparativos y superlativos). Todo ello, bien practicado y con la posibilidad de ser reelaborado en una posterior corrección y sistematización. En definitiva, un sistema de trabajo que implica un alto grado de conceptualización abstracta, de capacidad de reflexión sobre el lenguaje y de utilización de estrategias cognitivas como la organización o el contraste con la lengua materna.

\section{BASES PSICOLINGÜISTICAS PARA LA ENSEÑANZA/APRENDIZAJE DEL INGLES A LOS OCHO AÑOS}

Lo expuesto nos da ya varias pistas sobre qué tipo de criterios deben organizar los cursos de enseñanza temprana de una lengua extranjera. Empezaremos por un ejemplo. Eso nos permitirá ilustrar las bases para el diseño de actividades, la selección de temas y la secuencia de elaboración de los contenidos lingüísticos.

\section{Condiciones de diseño de actividades}

Si queremos enseñar a hablar y entender inglés deberemos ofrecer a nuestros alumnos actividades en las que el objetivo de las mismas no sea mostrar/ejemplificar el funcionamiento de unidades lingüísticas sino hacer cosas en inglés. Es decir, actividades en las que niños y niñas no son espectadores de lo que hacen unos personajes en inglés sino que son actores que deben usar el inglés para comunicarse. ¿Cómo? Veamos una muestra:

En este caso (Fig. 4) estamos introduciendo por primera vez los números. En el paso (a) proporcionamos un esquema conocido (once tarjetones con números) al que añadimos lenguaje desconocido. En los pasos (b) y (c) les obligamos a usar (comprender) los números de una manera significativa. Si no entienden, no pueden apuntar (o colocarse encima) al tarjetón con el número correcto. En el paso (d) los niños reconocen de nuevo los números al identificar los nombres de sus compañeros dando respuestas reales. Finalmente son guiados a usar los números para decir cosas verdaderas una vez más.

Las claves de la actividad pueden resumirse en: I. La participación activa de los niños en el uso del lenguaje. Desde el primer momento comprenden los números y los usan para hacer cosas (apuntar, colocarse encima y nombrar a quién está encima). Acaban produciendo los números para decir algo real (su edad) a alguien y no sólo para repetirlos. II. La sinceridad del uso del lenguaje. El inglés se usa para regular la actividad. Si no se usa bien, la actividad (el juego, los turnos, etc.) no sale. El inglés cobra todo su valor como instrumento y los niños aprenden a reconocer ese valor al contrastar los efectos de su uso en la 
FIGURA 4

Ejemplo de actividad (reproducido de Vale, 1990: Unit 1)

\section{TEACH NEW LANGUAGE}

Introduce the numbers $0-10$. Use flash cards of the numbers as teaching aids.

a. You show and say the numbers. The children LOOK and LISTEN ONLY.

$\mathrm{T}$ : This is number 1.

This is number 2. etc

b. The children point to the number (no speaking). You give the instructions.

T: Point to number 1.

Point to number 5. etc.'

c. The children play 'stand on the numbers'. You give the instructions.

T: Maria. Stand on number 7. Miki. Stand on zero. etc.

d. Ask the children to identify the numbers. You ask the questions. The children give SHORT TRUE responses.

T: Who's standing on number 6? Hands up. Ken, who's standing on number 7?

S: Maria.

T: Who's standing on number 10?

S: Me. etc.

T: Who's standing on number 8? Give me number 8 , please.

Thanks. Sit down, please. etc.

Continue until all the children are sitting down again.

e. Ask children how old they are. Use the «happy birthday» tune to explain your question.

T: How old are you Mariko?

S: Nine.

T: How old are you, Toni? etc.

actividad. No se habla de personajes de ficción o de cosas no presentes sobre las que la información transmitida mediante el lenguaje no produce un efecto perceptible. Si no se usa correctamente el inglés pasa algo directamente perceptible por los niños en la misma dinámica de la actividad. Si en lugar de apuntar al cinco apunta al siete, no se pasa el turno al siguiente (o se pasa pero se vuelve al que se ha equivocado para que lo haga otra vez, etc.). III. La creación de un contexto compartido (reconocido por todos) sobre el que se presentan las unidades de lenguaje desconocido. La actividad es un juego (varios juegos) y los niños son expertos conocedores de sus reglas. Saben cómo actuar en la actividad, sólo les faltan las piezas necesarias (el inglés) para poder resolverla. Hemos creado una estructura conocida por el niño que va a permitir anclar significativamente los nuevos elementos (los números en inglés) a algo que ya domina. IV. La creación de un motor afectivo para aprender lo que no saben. Lo hacemos si las actividades son divertidas y/o interesantes. El planteamiento en forma de juegos incita a los niños a resolverlos. Las reglas las conocen, la curiosidad por hacerlo bien les va a llevar a utilizar lo desconocido, el inglés y a dominarlo. Como resultado, el éxito de cada niño en su turno actúa de motor para una mayor implicación 
en la clase. El éxito en el uso del inglés es el éxito en la actividad y por tanto el motor del aprendizaje.

\section{Mecanismos de aprendizaje instrumental del lenguaje}

El ejemplo ilustra claramente la primera condición para que nuestros alumnos aprendan inglés. La enseñanza del inglés se basa necesariamente en la necesidad de crear situaciones de uso del lenguaje en el aula donde los aprendices puedan utilizar los contenidos del inglés para realizar acciones de comunicación.

Es decir, dicho en otros términos, una lengua se aprende cuando se usa. El problema se deriva de preguntarnos cómo se puede usar una lengua que no se conoce. Es decir, en términos de Artigal (1990), ¿cómo es posible que el aprendiz de una lengua extranjera utilice ésta para aprenderla si no la conoce?

Algunos autores han intentado arrojar algo de luz sobre esta cuestión. Krashen (1982) afirma que los aprendices usan el inglés desde el momento en el que son capaces de entender el significado de los mensajes inmersos en actividades de comunicación. Para Krashen, el «secreto» radica en la utilización de claves contextuales (gestos, objetos, dibujos, etc.) sobre las que apoyar la aparición de los elementos lingüísticos nuevos, de nivel «i $+1 »$ que, al ser entendidos extralingüísticamente (decir sit down/stand up mientras el profesor se sienta/levanta) pueden ser utilizados (comprendidos) para la comunicación.

En este caso, Krashen nos está llamando la atención sobre la importancia de la comprensión de las actividades en lengua extranjera. De esta manera, no sólo los elementos linguísticos puntuales deben ser objeto de un tratamiento didáctico (mímica, imagen, objeto, etc.) que garantice su comprensión, sino el «significado» de la misma tarea, entendida como una actividad cooperativa debe ser también compartido. Es decir, además, el objetivo de la tarea (jugar a X/cantar/simular/describir elementos/explicar-entender/etc.), los roles de los que intervienen (escuchar/repetir/participar en turnos/etc) y la estructura de la actividad (principio/pasos/fin/etc.) deben ser entendidas (explícita o implícitamente) por los aprendices.

Otros autores (Swain, 1985; Ervin-Tripp, 1987) reivindican, junto con la comprensión, la importancia de la producción de inglés como condición para su aprendizaje. En este caso, la producción en inglés permite I) la producción de mensajes en inglés de un nivel superior a la capacidad individual gracias al contexto cooperativo de la actividad que se está desarrollando (el sistema de turnos de oir la instrucción y apuntar), y II) la utilización de las muestras en inglés producidas como instrumento para la significación de otros (o las mismas) mensajes/elementos presentes al contrastarlos en el contexto de la actividad.

Es decir, el aprendizaje del inglés requerirá oportunidades de producir inglés significativamente. La creación de actividades donde los aprendices participen en inglés implicará la elaboración de un contexto compartido sobre el que anclar los significados de muestras en inglés que no se conocen pero que señalan aspectos de la actividad en curso.

Este anclaje en la comprensión de la estructura de la actividad no es sólo por sentido común. Al plantear un juego como el de los números, o al hacer más adelante que los alumnos se midan con un metro, o al ordenar y pegar en el cuaderno los dibujos de lo que ya sabemos en inglés en grupos (animals, instruments, numbers, food, etc.) estamos permitiendo que el alumno utilice lo que ya sabe para aprender cosas nuevas. 
Dicho en términos psicológicos, al crear un contexto de actividad reconocible por el alumno activamos sus esquemas de conocimiento (Coll, 1983) de ese contexto. Si le hacemos trabajar en él en inglés significativamente, cuando la actividad se acabe, el esquema de conocimiento volverá a la memoria profunda del niño, pero regresará cargado con nuevo conocimiento, en este caso de inglés. Estas nuevas unidades de conocimiento se hallan ligadas al uso real del lenguaje ya que así ha sido conocido por primera vez, asegurando su dimensión instrumental.

Lógicamente, esto implica una selección de actividades teniendo en cuenta el mundo del niño y olvidándose de todo aquello que no sea vinculable a la experiencia de actividad del mundo infantil a los ocho años. De esta manera, las mejores actividades serán las que reproduzcan el mundo experiencial del niño de ocho años, sus juegos, sus temas de interés, sus rutinas académicas, sus experiencias y materias en la escuela. Pero en inglés.

Este proceso de activación de esquemas, enriquecimiento con unidades nuevas en el curso de la actividad y almacenamiento nos permite explicar el aprendizaje instrumental del inglés. ¿Cómo? Mediante lo que Artigal (1990), desde una perspectiva semiótica, caracteriza como naturaleza indexical de los primeros usos del inglés. La naturaleza de los significados de las unidades lingüísticas del inglés comprendidas/producidas por los niños en estos primeros usos altamente contextualizados nos lleva a preguntarnos sobre la definición de los significados construidos. ¿Qué significan para el infante los primeros signos/unidades de inglés que aparecen en las tareas de aula?

La definición del lenguaje como un conjunto de signos (Silverstein, 1985) nos remite a establecer diferentes tipos de relaciones entre los signos y los objetos a los que se refieren. Desde esta perspectiva, es posible distinguir entre valor indexical y valor simbólico de los signos lingüísticos. El valor indexical de un signo viene dado por la copresencia con el objeto al que se refiere (sit down indica la acción que coocurre con su producción), mientras que su valor simbólico lo hace independiente espacio-temporalmente del objeto referido (sit down como significado de la acción imperativa del verbo sentarse). Mientras que los valores indexicales son efímeros por «significar» sólo en cada contexto en el que aparecen, los valores simbólicos son convencionales, y corresponden a los significados permanentes de los signos en una comunidad lingüística determinada.

La hipótesis mantenida desde este planteamiento implica que los primeros significados de los signos inglés construidos por el aprendiz son de naturaleza indexical y sólo después pasan a ser simbólicos. La aparición en un contexto de uso de un signo - no conocido- «right han up» indica (en este caso por oposición al enunciado «left band up») un valor indexical - dado por la acción copresente - que permite la elaboración de un primer significado de la producción. El término (o elemento) right/left, al aparecer en otro contexto (right? mirando a la mano y preguntando al profesor o turn right a otro alumno en un juego en el aula donde el otro hace de robot), recibirá un nuevo valor indexical que, combinado con el anterior permitirá construir un significado más cercano a su valor simbólico (orientación respecto a un eje vertical).

De esta manera, al pasar a actividades y temas nuevos donde son usadas unidades lingǘsticas que ya han sido utilizadas anteriormente, creamos nuevos estratos de significación en este recorrido del valor indexical al valor simbólico. El final podrá ser un dominio también conceptual de las unidades lingüísticas. Pero nunca el comienzo como pasaba con los cursos analizados al inicio de este 
trabajo. Mediante este proceso de aparición y diversificación contextual se irá produciendo una progresiva elaboración del carácter simbólico del material lingüístico. Su culminación se producirá cuando el mismo lenguaje (inglés) se convierta en contexto de indexicalización y simbolización posterior. Es decir, cuando apliquemos índices lingüísticos a unidades de inglés. Por ejemplo, al agrupar un grupo de palabras bajo la familia food, o numbers, o al sistematizar la regla de cuando se pone o deja de poner el auxiliar $d o$.

\section{La secuencia de elaboración del conocimiento lingüístico}

Este proceso configura una secuencia de elaboración de los contenidos muy diferente a la que presentan los cursos analizados (recordemos: presentación, aprendizaje, ejercitación). La secuencia de construcción de significados de ingleś deberá pasar por cuatro fases diferenciadas: I) una fase inicial de uso (comprensión/producción) contextualizado de los contenidos del inglés donde a partir de la comprensión conjunta de todos los elementos (objetivo, acciones, estructura, objetos, roles, etc.) se anclen los contenidos de inglés objeto de aprendizaje; II) una segunda fase de reaparición y producción de inglés en el contexto de otras actividades donde se diversifiquen/reelaboren/comprueben los primeros significados elaborados; III) una tercera fase de generalización/simbolización que permita integrar/sistematizar los nuevos significados en el conocimiento de los niños; y IV) una fase de reaparición posterior y reutilización con otro material nuevo o vinculado al ya conocido.

Volviendo al análisis inicial de los cursos de inglés se trata de romper con el error de asumir en los niños la capacidad adulta de organizar mentalmente cada bloque de contenidos por separado y guardarlos para volver a utilizarlos cuando sean requeridos. La secuenciación de contenidos en estos cursos es lineal, cuando un contenido ha sido visto y repasado ya no vuelve a aparecer hasta el curso siguiente, dando secuencias como la del ejemplo:

FigURA 5

$\begin{array}{lccc}\text { TOTAL HORAS CURSO } & 50 \% & & 100 \% \\ 1 & 45 & 66697277 & 90 \\ \text { UNIDADES DIDACTICAS } & 15 & 22232425 & 30 \\ 1 & & * * * * & \\ & & *\end{array}$

Secuencia de aparición de los contenidos RIGHT/LEFT en las actividades de un curso comunicativo

Donde en un curso de un total de 90 horas, los contenidos right/left aparecen como objeto de trabajo en la unidad 22 (a las 66 horas) mediante dos actividades diferentes (una de presentación y otra de aprendizaje), en las unidades $23 / 24$ en sendas actividades de aprendizaje y en la unidad 25 en una actividad de repaso clásica. Algo que desde nuestro punto de vista tiene poca efectividad. 
Esto, necesariamente, nos lleva a una nueva condición. El curso debe garantizar una reutilización progresiva del inglés trabajado en cada unidad didáctica. Esta no puede ser entendida como un repaso o confirmación de lo bien aprendida que está como veíamos hace unas líneas. Queremos decir volver a crear un contexto donde lo ya aprendido sea utilizado para hacer otras cosas. Los números serán utilizados para poner etiquetas en cajas en la siguiente unidad didáctica, para medir a los alumnos y anotar sus alturas en un cuadro dos unidades más allá, para ser recortados (escritos con letras) y agrupados bajo el título numbers cinco unidades después, etc. De esta manera, cada vez más el conocimiento de esas unidades lingüísticas se enriquece y posee más contextos de activación/utilización. Incluso el más formal, el que los conceptualiza como categoría léxica.

\section{LA ENSEÑANZA DEL LENGUAJE MEDIANTE TAREAS}

Las implicaciones educativas de esta aproximación a los procesos psicolingüísticos implicados en el aprendizaje de una lengua extranjera son numerosas.

\section{Implicaciones educativas de un análisis psicolingüistico}

La adopción de los criterios expuestos implica que el sistema de trabajo empleado debe: utilizar técnicas didácticas que permitan crear una dinámica en el aula donde mientras que todo se hace en inglés los niños son capaces de entender lo que está pasando y pueden seguir el desarrollo de las actividades paso a paso; diseñar las actividades de manera que niños y niñas puedan entender el inglés que aparece como vehículo para las instrucciones, o para nombrar las cosas o para reír y crear situaciones afectivas y divertidas; diseñar actividades donde los niños puedan ser conducidos a que hablen progresivamente ne inglés con las condiciones antes mencionadas de sinceridad y éxito en sus intervenciones; diseñar actividades generadas por temas vinculables al mundo experiencial del niño y, por tanto, motivantes e interesantes afectivamente; v. Generar actividades de aprendizaje del lenguaje con el objetivo de introducir progresivamente los contenidos para que los niños los utilicen (los entiendan y los produzcan) dentro de las actividades, permitir la reaparición cíclica de la utilización de estos contenidos en otras actividades tanto dentro de la unidad como a lo largo del curso y organizar periódicamente actividades de corrección, reorganización y sistematización del material aprendido.

El diseño de la enseñanza del inglés parece, por tanto, más susceptible de articularse en torno a las tareas que guiarán este proceso que a los contenidos objeto de aprendizaje. De esta manera será posible diferenciar tipos de tareas relacionados con su implicación en cada una de las fases descritas. Su articulación y secuenciación deberá hacerse conforme a los objetivos de cada unidad didáctica mediante un plan de elaboración que garantice la llegada a cada bloque de objetivos.

\section{Los sistemas de actividades o tareas para la enseñanza del inglés}

Algunas propuestas (Curtain y Martínez, 1990; Estaire y Zanón, 1990, Vale, 1990; Williams, 1991) especifican el proceso a seguir para la elaboración de cursos de enseñanza de inglés mediante tareas o actividades. Todas comparten tres características que consideramos fundamentales: 
I. Las unidades didácticas se generan desde temas de interés de los niños y no desde contenidos lingüíticos. II. La elaboración de las lecciones que componen una unidad didáctica toma como unidades las actividades a hacer por los niños en inglés adecuándose a los criterios recién expuestos; y III. El control de los contenidos lingüísticos trabajados se ejerce mediante la elaboración de un "syllabus retrospectivo» que permite ajustar progresivamente el curso.

En todas estas propuestas, el punto de partida será siempre un tema (Christmas, animals, holidays, etc.) directamente relacionado con el mundo experencial de los niños y que interesará y motivará la realización de las actividades.

El resultado puede ser un curso cuyo diseño nada tendrá que ver con los clásicos cursos basados en contenidos:

Figura 6

Syllabus de un curso basado en actividades (reproducido de Vale, 1990)

\section{CONTENTS}

1. The children make a passport page

2. The children display their photos

3. The children play Buzz and Beetle

They also practise multiplication in English

4. The children sort and life items into their picture dictionary

5. The childen make a snapdragon

6. Andy and Annie. Cartoon story time

7. The Children make a "join the dots "picture"

They also find facts about the elephants

8. The children sort and file items into their picture dictionary

9. The children make a 'favorites'crest

10. The children make a face mask based on the shape of a friend's face

14. The children interview classmates

16. The children sort and sequence numbers and sizes

They also do work on comparisons

17. The children make a collage

18. The children study a street scene.

23. Story time

25. The children build up sets

En el ejemplo, las unidades didácticas son actividades finales (hacer un collage o medirse y hacer una tabla de alturas) que generan toda una serie de actividades previas que constituyen lecciones organizadas en torno al tema de la actividad. Nunca en torno a un conjunto de contenidos lingǘsticos.

El despliegue del tema en actividades puede hacerse de diferentes maneras. Williams (1991), propone un marco para la elaboración de unidades mediante actividades generadas por un tema.

El proceso a seguir consiste en un primer estadio (Fig. 7) donde se generan todas las actividades posibles en torno a un tema determinado para en un segundo estadio organizarlas y secuenciarlas conforme a un conjunto de criterios análogos a los expuestos. El resultado es un conjunto de lecciones cuyas actividades (oír y explotar un cuento, hacer un sombrero de bruja, etc.) determinan el avance por una serie de aspectos en relación al tema escogido (Halloween) 
Figura 7

Primer estadio en la elaboración de una unidad didáctica basada en actividades (reproducido de Williams, 1991)

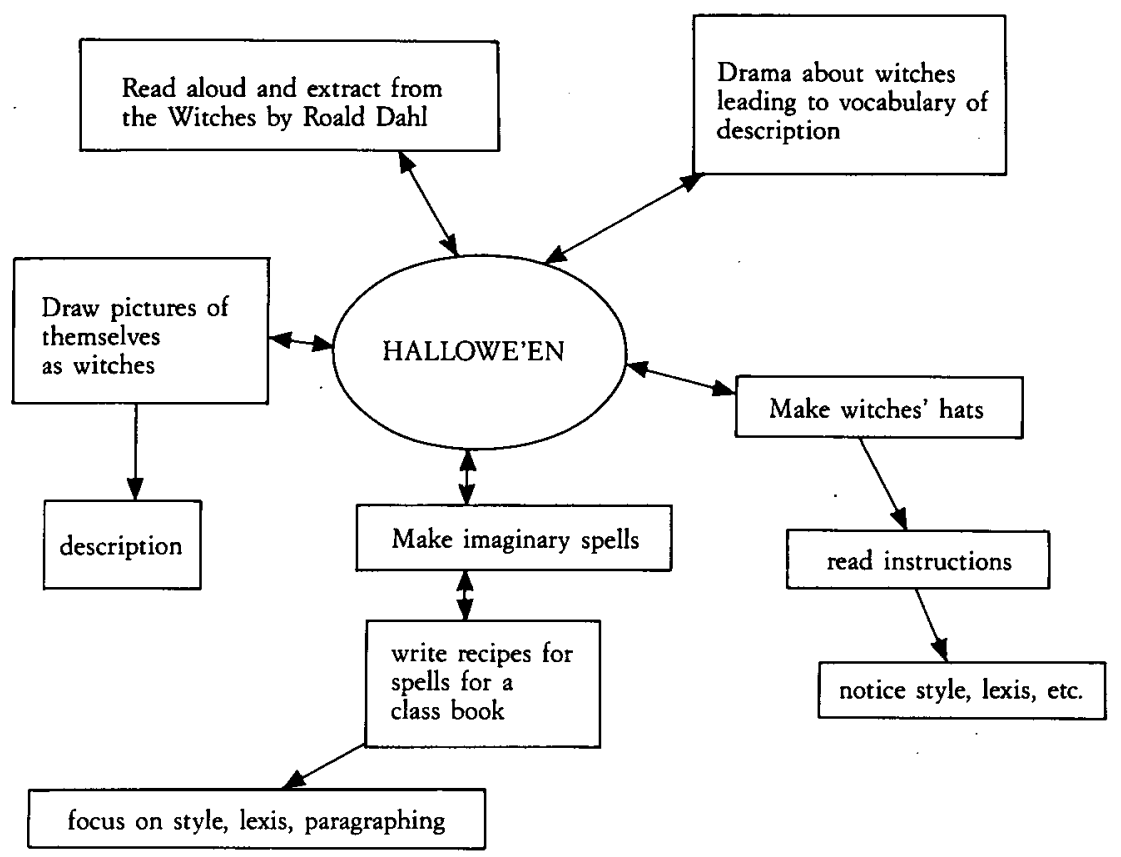

y cuyo trabajo requiere la preparación y aprendizaje de un conjunto de contenidos lingüísticos (estructuras, nociones, funciones, etc.).

De forma parecida (aunque para enseñanza secundaria) el diseño de unidades didácticas mediante tareas (Estaire y Zanón, 1990) se muestra como un marco útil para el diseño de la enseñanza precoz del inglés ya que permite el desarrollo del trabajo con el inglés a partir de la definición de un tema o área de interés no lingüístico (la familia, la casa, la calle, la escuela, la clase, etc.), sobre el que anclar los objetivos de la unidad y que permitirá dar sentido a los contenidos lingüísticos a trabajar; asegurar la partida del trabajo en inglés de la realidad experiencial de los aprendicezs; su desarrollo a través de otras áreas del currículum como la expresión plástica; la incorporación de bloques de objetivos y contenidos paralelos a los del área de lenguaje para la educación primaria del DCB (MEC, 1989), y la evaluación incorporada como parte del proceso de enseñanza/aprendizaje al tomar las tareas como punto de referencia del avance de la unidad.

El proceso a seguir es similar al anterior pero con un elevado grado de estructuración. Los seis pasos del marco (Fig. 8) determinan unidades formadas por tareas que, de nuevo, han surgido dé un tema no lingüístico de interés para los alumnos. La novedad, en este caso, es la definición de una(s) «tarea(s) final(es)» que en forma de actividad comunicativa representan el punto de llega- 


\section{6}

Figura 8

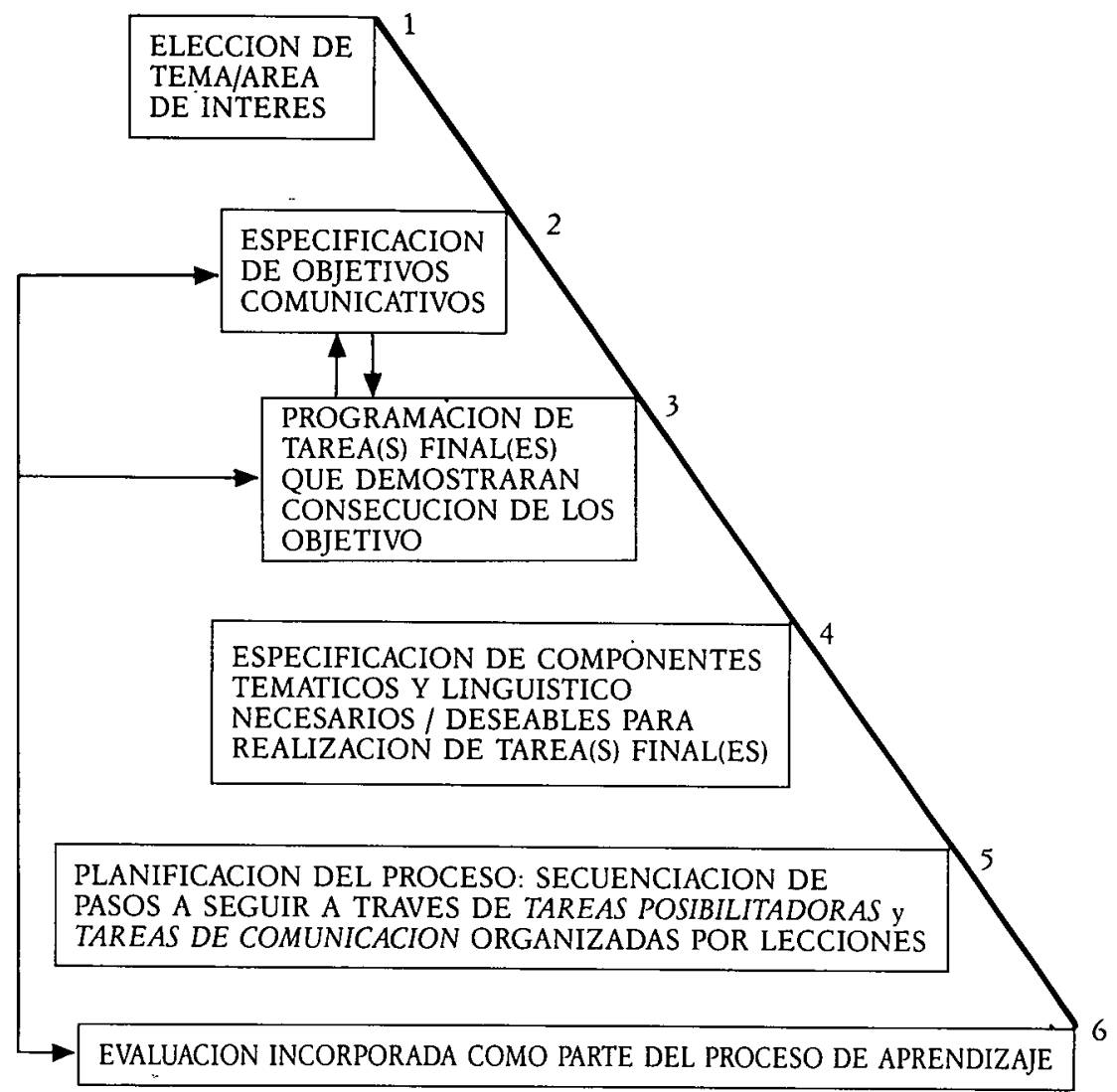

Marco para el diseño de unidades didácticas mediante tareas. (reproducido de Estaire y Zanón, 1990)

Marco para la programación de una unidad didáctica a través de tareas (integrando objetivos, contenidos, metodología y evaluación).

da de la unidad y que generarán las necesidades en forma de otras tareas posibilitadoras y de comunicación, de aspectos del tema a tratar, de contenidos lingüísticos a trabajar, etc.

\section{El syllabus retrospectivo}

El desarrollo del syllabus de contenidos se hace en estos casos mediante un «syllabus retrospectivo», es decir, un catálogo minucioso de los diferentes bloques de contenidos que se han tratado a lo largo de la unidad. Mediante su segiminto puede determinarse la necesidad de ciertas actividades en unidades posteriores o la planificación de las unidades siguientes o del curso del año próximo.

Esto toma especial importancia dado el carácter cíclico y no lineal del aprendizaje del lenguaje que se desprende del análisis expuesto (ver ap. 3). La concepción de un conjunto de contenidos que «uno más uno» van proporcionando el conocimiento de la materia debe dejarse para áreas de conocimiento con ele- 
vada carga conceptual. En todo caso nunca para enseñar algo tan instrumental y dinámico como el lenguaje.

El trabajo con un syllabus retrospectivo es especialmente adecuado en nuestro contex to educativo. Su aplicación (Estaire y Zanón, 1990) permite elaborar un mapa unidad a unidad de los diferentes tipos (conceptos, principios, procedimientos, etc.) y bloques (expresión oral, comprensión oral, etc.) contemplados en los diseños curriculares del área de lenguas extranjeras (MEC, 1989).

\section{El valor educativo de aprender a hablar inglés}

En las tres propuestas comentadas destaca el valor educativo que la enseñanza del inglés puede tener desde un diseño mediante actividades. En todas ellas, niños y niñas son llevados a hacer cosas en la clase de inglés.

Esto beneficia a los alumnos en tres aspectos clave: I. El trabajo de contenidos de otras áreas de forma diferente. Desde actividades de plástica a juegos lógicos y de cálculo numérico, pasando por todo tipo de actividades de expresión y comunicación. II. El desarrollo de comportamientos sociales. La dinámica de unas actividades donde hay que decir cosas a alguien para algo sugiere un claro énfasis en el trabajo cooperativo, la toma de decisiones; el respeto mutuo, etc.; y III. El desarrollo cognitivo propiciado por la incorporación instrumental del inglés. Un instrumento generador de pensamiento abstracto al facilitar la categorización y el contraste de formas con la lengua materna.

\section{PROBLEMAS Y PERSPECTIVAS}

Estamos siendo testigos de un enorme auge de la enseñanza del inglés a los ocho años. El desarrollo de la reforma de nuestro sistema educativo es en buena medida responsable de ello. La nueva estructura curricular determina que en nuestras escuelas se empiece a enseñar inglés a niños y niñas de ocho años.

Sin embargo, el despliegue masivo de esta medida puede dar al traste con los avanzados objetivos del área de lenguas extranjeras del proyecto curricular. La enseñanza del inglés a los ocho años debe cumplir un conjunto de condiciones derivadas de un análisis psicopedagógico de los procesos de aprendizaje de una lengua extranjera en esta edad que se combinan con las condiciones propias del trabajo en un sistema educativo «madre».

En este sentido, la introducción temprana de la lengua extranjera (LE) en el currículum escolar puede caracterizarse como un proceso de toma de decisiones sobre tres niveles del diseño educativo (Zanón, 1990). El primer nivel (A) corresponde al del programa general del ciclo de EGB donde se comienza la enseñanza de la LE. Las decisiones a tomar aquí determinarán la edad de introducción de la LE, la cantidad de horas a la semana dedicadas a su enseñanza, las áreas curriculares implicadas y su desarrollo a lo largo de los cursos posteriores. Un segundo nivel (B) define el programa de LE propiamente dicho. Esto implica la definición de los objetivos, los contenidos, el planteamiento didáctico y los recursos y materiales a utilizar. Finalmente, el tercer nivel concreta en forma de actividades de aula las intenciones educativas desarrolladas en los niveles $A$ y $B$. En este punto, el/la profesor/a se enfrenta a la definición de los tipos de actividades a desarrollar con los contenidos seleccionados, a su articulación y secuenciación en el camino hacia los objetivos prefijados y a su posterior evaluación. 


\section{8}

Como es fácil ver, este proceso es una labor delicada que requiere un poderoso bagaje técnico. La situación actual del profesorado no parece ser la idónea para asumir un sistema como el descrito en este trabajo. Las condiciones de partida para esta tarea desde nuestro contexto educativo son claramente inadecuadas, poniendo en peligro no sólo el futuro contacto de los alumnos con el inglés sino el empeño e ilusión de muchos profesores del área.

Los escollos a superar derivados del análisis que hemos hecho aquí pueden resumirse en:

I. El nivel de inglés de los/as profesores/as de inglés. Para enseñar a hablar inglés hay que hacerlo en inglés. Esto no quiere decir que los/as profesores/as deban ser nativos. Pero sí que sin un mínimo dominio fluido del inglés sea prácticamente imposible gestionar las actividades dentro del aula. Sin este requisito previo difícilmente se elevará el nivel de calidad de la enseñanza del inglés.

II. La concepción del aprendizaje del inglés de los/as profesores/as. Con una obstinación casi religiosa parece difícil persuadir a los/as profesores/as de que el lenguaje no se aprende unidad a unidad. La necesidad de romper con la creencia en un syllabus lineal de contenidos lingüísticos como base de un curso de inglés es otro requisito previo para el cambio hacia un sistema como los descritos.

III. Las necesidades de formación en diseño de actividades. La adopción de un sistema de trabajo basado en actividades implica saber diseñar actividades. Esto requiere una formación específica en varias áreas clásicas de la didáctica del inglés. Desde diseño de actividades específicas para cada «skill» (hablar, comprender, leer y escribir) al de juegos, explotación de materiales, etc.

IV. Las necesidades de formación en técnicas de corrección, etc., implica el empleo de técnicas de aula. El trabajo en una lengua extranjera con niños requiere un conjunto de técnicas didácticas específicas. Asegurar el ambiente lúdico, la comprensión de todo lo que pasa, las estrategias para incitar a los alumnos a hablar, las técnicas de aula como el «Total Phisical Response» o la mímica necesarias para el trabajo con niños.

V. El diseño de cursos mediante actividades. El sistema propuesto implica dominar el marco de diseño de cursos mediante actividades. Esto sólo puede hacerse mediante un asesoramiento de especialistas que, en los primeros momentos, puedan enseñar a los/as profesores/as a solucionar los problemas derivados de la novedad del sistema.

VI. Los recursos del profesor y el centro. Trabajar al nivel descrito implica una profesionalización obvia. Esto requiere ciertas condiciones materiales que pasan por el número de alumnos en las aulas, el número de horas de docencia de los/as profesores/as, el acceso a materiales didácticos del centro, etc.

Las conclusiones que se derivan de este análisis pueden parecer desalentadoras. No es así. Son solamente una precaución. Quizá valga la pena reflexionar un poco antes de actuar. Sin duda si somos capaces de asumir nuestras propias necesidades, intentaremos crear las condiciones para satisfacerlas. En suma, se trata de asumir un compromiso profesional. Si no, caemos en el riesgo de engañar a nuestros clientes: los niños. Ellos nos lo agradecerán. 


\section{Referencias}

Artigst.J. M. (1990). El context compartit com a base de l'us/adquisició de la nova llengua. Manuscrito sin publicar.

CoLL.C. (1983). La construcción de esquemas de conocimiento en situaciones de enseñanza/aprendizaje. En C. Coll (ed.), Psicología Genética y Aprendizajes Escolares. Madrid: Siglo XXI.

Concari, M., et al. (1983). Snap! Londres: Heinemann.

Curtain, H. A. Y Martinez, L. s.(1990). Elementary School, Content-Based Foreign Language Instruction. En A. Padilla, H. Fairchild y C. Valadez (eds.) Foreign Language Education. Newbury Park, CAL: SAGE.

ERVING.TRIPP, S. (1987). «Activity structure as scaffolding for children's second language learning". En Cook-Gumperz, J., y Corsario, W. (eds.), Issues in Theory an Methods of Studying Children's Worlds. La Halle: Mouton.

Estaire, S., Y J. Zanón. (1990). El diseño de unidades didácticas mediante tareas. Principios y desarrollo. Comunicación, Lenguaje y Educación, $7 / 8$.

Krasien, S. D. (1982). Principles and Practice in Second Language Acquisition. Oxford: Pergamon Press.

Mєc. (1989). Diseño Curricular Base: Enseñanza Primaria.

SilverSTEIN. M. (1985). The functional stratification of language and ontogenesis. J. V. Wertsch (ed.), Culture, communication and cognition. Vygotskian Perspectives. Cambrigde. CUP.

Strange. D. (1989). Chatterbox. Oxford: O. U. P.

Swin. M. (1985). «Communicative Competence: somo roles of comprehensible input and comprehensible output and its development». En Gass, S., y Madden, C. (eds.) (1985). Input in Second Language Acquisition. Rowley, Mass.: Newbury House.

Trim. J. L. M. (1973). Programme de recherche et de mise au point d'un système europeèn d'unitées capitalisables pour l'apprentisage des langues vivantes par les adultes: raport d'activité. Consejo de Europa, CCC/EES (73), 26.

VALE. D. (1990). Early Bird. Teacher's Book. Cambridge: Early Bird Publishing.

VAN EK. J. A. (1975). The Threshold Level. Consejo de Europa.

WILLIAMS, M. (1991). A framework for Teaching English to young learners. En C. Brumfit, J. Moon y R. Tongue, (eds.), Teaching English to Children. Londres: Collins.

ZnNóN.J. (1988/89). Psicolingǘstica y didáctica de las lenguas: una aproximación histórica y conceptual. Partes I/II. Cable, 2/3.

Z ¿nÓN. J. (1990). Aspectos Psicolingüísticos de la enseñanza/aprendizaje de una lengua extranjera en edades tempranas. Signos, 1. 


\section{Cómo no impedir que los niños} aprendan inglés

\section{Javier Zanón}

CL\&E, 1992, 16, pp. 93-110

Resumen: La mayoría de materiales disponibles para la enseñanza de las lenguas extranjeras en la escuela primaria muestra una estructura didáctica poco adecuada desde un punto de vista psicopedagógico. En ellos se mantiene una concepción de la lengua como contenidos y se estructura el trabajo en las unidades como un proceso que asegure el dominio de esos contenidos lingüísticos. En este trabajo, partiendo de una concepción instrumental de la lengua se propone una fundamentación psicolingüística del aprendizaje de la lenguja extranjera que conduce aun sistema basado en actividades para el diseño de unidades didácticas y cursos de inglés. Finalmente, se reflexiona sobre las implicaciones que puede tener adoptar un sistema basado en actividades para el profesorado.

Datos sobre el autor: Javier Zanón trabaja como investigador en el área de lenguas extranjeras en el Institut de Ciències de l'Educació de la Universitat de Barcelona.

Dirección: Instituto de Ciencias de la Educación de la Universidad de Barcelona. Gran Vía Corts Catalanes, 585. 08007 Barcelona.

Agradecimientos: Una versión informal de este documento fue utilizada como guión para impartir el curso «The psychopedagogical basis for the teaching of EFL to 8-10 year old children» dentro del ciclo de seminarios para especialistas sobre la enseñanza del inglés a niños y niñas. Teacher Development Unit. The British Council. Madrid, febrero de 1991.

Quiero agradecer especialmente la colaboración de los organizadores y participantes en el curso citado y en el siguiente seminario impartido por Marion Williams. Sin sus comentarios, dudas y discrepancias este trabajo nunca habría visto la luz.

(C) De todos los artículos deberá solicitarse por escrito autorización de CL\&E y de los autores para el uso en forma de facsímil, fotocopia o cualquier otro medio de reproducción impresa. CL\&E se reserva el derecho de interponer las acciones legales necesarias en aquellos casos en que se contravenga la ley de derechos de autor.

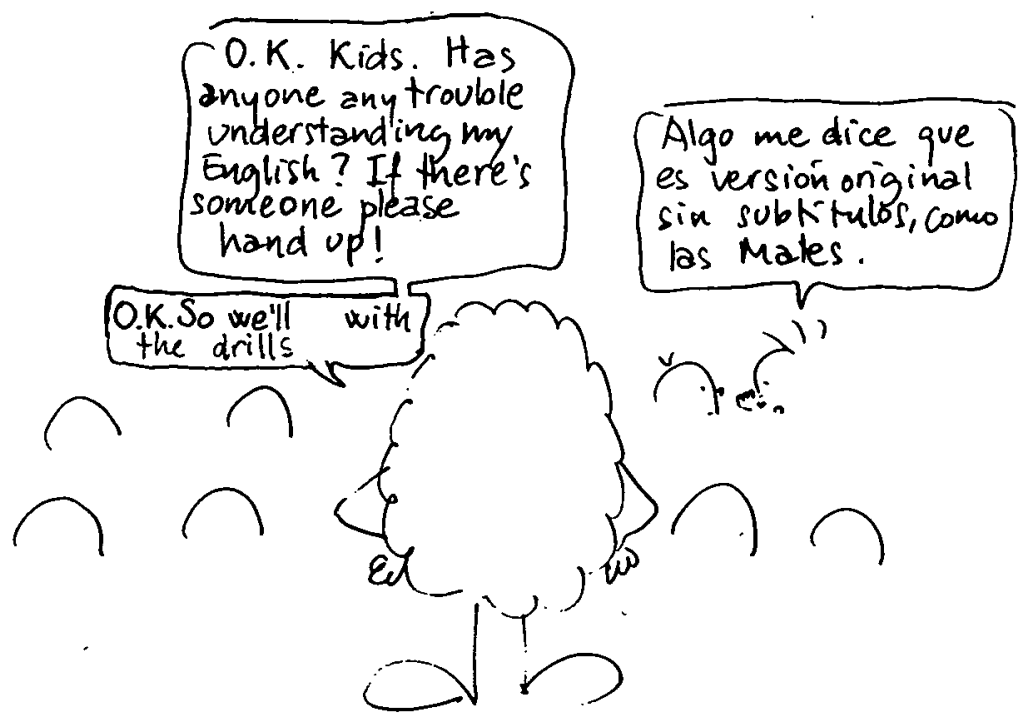

